\title{
A search for rapid optical variability in radio-quiet quasars
}

\author{
M. Rabbette ${ }^{1}$, B. McBreen ${ }^{1}$, N. Smith ${ }^{2}$, and S. Steel ${ }^{1}$ \\ 1 Physics Department, University College, Dublin 4, Ireland \\ 2 Applied Physics and Instrumentation Department, Regional Technical College, Cork, Ireland
}

Received May 5; accepted October 7, 1997

\begin{abstract}
The detection of rapid variability on a timescale of hours in radio-quiet quasars (RQQSOs) could be a powerful discriminator between starburst, accretion disc and relativistic jet models of these sources. This paper contains an account of a dedicated search for rapid optical variability in RQQSOs. The technique used differential photometry between the RQQSO and stars in the same field of view of the CCD. The 23 RQQSOs that were observed all have high luminosities $\left(-27<M_{V}<-30\right)$, and 22 of these sources are at redshifts $z>1$. The total amount of observation time was about 60 hours and these observations are part of an ongoing programme, started in September 1990, to search for rapid variability in RQQSOs. No evidence for short-term variability greater than about 0.1 magnitudes was detected in any of the 23 sources, however long-term variability was recorded for the radio-quiet quasar PG $2112+059$. The finding charts are included here because they identify the RQQSO and the reference stars used in the photometry, and hence are available for use by other observers.

The unusual properties of two RQQSOs that were not included in our source list are noted. X-ray results reveal that PG $1416-129$ is variable on a timescale of days and that the remarkable source IRAS $13349+2438$ varied by a factor of two on a timescale of a few hours. The latter source displayed blazar type behaviour in X-rays and implies that relativistic beaming may occur in at least some RQQSOs. Radio results also indicate the presence of jets in at least some RQQSOs.
\end{abstract}

Key words: galaxies: active — galaxies: photometry quasars: general

\section{Introduction}

There is a general consensus that quasars belong to two different radio populations, radio-quiet quasars (RQQSOs) and radio-loud quasars. $R$ is usually defined as the ratio of the radio $(6 \mathrm{~cm})$ to the optical $(440 \mathrm{~nm})$ flux densities and the radio-quiet quasars have a value of $R<10$, while the radio-loud quasars have $R>10$ (Kellermann et al. 1989). It is found that $\sim 10 \%$ of quasars are in the radio-loud category. An additional distinction between active galactic nuclei (AGN) with strong and weak radio sources comes from the observation that radio loud objects essentially all occur in elliptical galaxies and RQQSOs appear to reside in galaxies that are dominated by exponential disks. However the RQQSOs that occur in elliptical host galaxies are in general more luminous than those that reside in disks (Taylor et al. 1996).

Little is known about the short-term variability of radio-quiet quasars, because few studies have been carried out (Gopal-Krishna et al. 1993 and 1995; Jang \& Miller 1995; Sagar et al. 1996). In contrast blazars display rapid variability in the wavelength range from radio to gamma rays. The blazar class encompasses both optically-violently-variable (OVV) quasars and BL Lac objects and about one quarter of all radio-loud quasars are also in the blazar category (Webb et al. 1988; Pica et al. 1988).

There are many theoretical models which endeavour to explain the large and rapid variability exhibited by blazars and these are usually divided into extrinsic and intrinsic categories. One extrinsic mechanism is microlensing of emission knots in a relativistic jet when they pass behind planets in an intervening galaxy (McBreen \& Metcalfe 1987; Gopal-Krishna \& Subramanian 1991). The rapid variability from superluminal-microlensing may be responsible for the variability observed in AO $0235+164$ (Rabbette et al. 1996) and PKS 0537-441 (Romero et al. 1995). One family of intrinsic models is based on a rotating supermassive black hole which accretes matter from a surrounding accretion disc and ejects two oppositely directed jets. The shocked-jet model involves shocks which move with relativistic speeds along the jet (Qian et al. 1991; Marscher 1980). It is believed the shock propagates along the line of sight, through inhomogeneous, small-scale structures distributed along the jet. These inhomogeneous structures are illuminated, or excited, by the moving relativistic shock, through the amplification of the magnetic field and the acceleration of electrons which causes the 
variability in polarization and in flux density that are observed over a wide range of frequencies (Hughes et al. 1986). Another family of intrinsic models invokes numerous flares or hotspots in the accretion disk and the corona that is believed to surround the central engine (Wiita et al. 1992; Mangalam \& Wiita 1993) and indeed a similar model has been proposed to explain X-ray variations in blazars (Abramowicz et al. 1991). The fact that RQQSOs generally lie on the far-infrared versus radio correlation (Sopp \& Alexander 1991) suggest that star formation plays an important role in their radio emission. It has been suggested by Terlevich et al. (1992) that the low values of $R$ in RQQSOs can be explained without jets or accretion discs, by postulating a circumnuclear starburst within a dense, high-metallicity nuclear environment. In this model the optical/UV and bolometric luminosity arises from young stars; the variability comes from cooling instabilities in the shell of compact supernova remnants and supernova flashes. Variability on intranight timescales is however difficult to explain with this model because of the short timescales involved. Furthermore radio-quiet and radioloud quasars have very different radio power outputs but have similar spectral shapes in the radio region and suggest that a significant fraction of the RQQSOs may be capable of producing powerful radio emission (Barvainis et al. 1996). Kellermann et al. (1994) found possible radio extensions up to about $300 \mathrm{kpc}$ in a few RQQSOs and assert that for at least these few cases, the emission is too large to be starburst related (Stein 1996).

Recently, some evidence suggesting rapid optical variability in the RQQSOs PG $0946+301$ and PG $1444+407$ was reported by Sagar et al. (1996). They also reported long-term variability for four RQQSOs. Jang \& Miller (1995) reported intranight variability for one RQQSO out of a sample of nine sources. Brinkmann et al. (1996) obtained ASCA observations of the radio-quiet, infrared quasar IRAS $13349+2438$ and detected substantial X-ray variability on a timescale of only a few hours.

The results of the photometric observations of a sample of mainly high luminosity and high redshift RQQSOs are presented. The observations and data reduction are given in Sect. 2. The results including tables listing the differential photometry and some light curves are presented in Sect. 3. The discussion and conclusions are given in Sects. 4 and 5. Section 4 also includes a discussion on two remarkable RQQSOs, PG 1416-129 and IRAS $13349+2438$. CCD images of the fields containing the radio-quiet quasars and reference stars used in the differential photometry are also included. A value of $H_{0}=50 \mathrm{~km} \mathrm{~s}^{-1} \mathrm{Mpc}^{-1}$ and $q_{0}=0.5$ has been adopted.

\section{Observations and data reduction}

The radio-quiet quasars were selected from the catalogues of Véron-Cetty \& Véron (1985); Hewitt \& Burbidge (1987) and Irwin et al. (1991). All the sources are at high red- shifts $(z>1)$ with the sole exception of PG $2112+059$ $(z=0.466)$ and the majority of the sources also have high absolute magnitudes $\left(-27<M_{V}<-30\right)$. The photometric data presented here were obtained during a number of observing runs spanning a period of six years. The observations were carried out during September 1990, October/November 1991, August 1992, September 1992, February 1993, March 1994, December 1995 and May/June 1996. All the RQQSOs listed in Tables 1 and 2 , were monitored during at least one of these observing periods between 1990 and 1996 and a few were observed over a number of years.

The observations were made with the one metre JK Telescope at the Observatorio del Roque de los Muchachos. A GEC CCD with $380 \times 580$ pixels was used for the September 1990 and October/November 1991 runs. An EEV CCD with $1246 \times 1152$ pixels and an image scale of 0.30 arcseconds pixel ${ }^{-1}$ was used for all the subsequent observing runs. The latter CCD was preferred because the larger field of view offered a greater choice of reference stars for the differential photometry. The seeing typically varied between 1.0 and 1.8 arcseconds.

The CCD fields containing the sources were observed through $B, V$ or $R$-band filters and integrations times varied between 3 and 14 minutes. The simultaneous observations of the source and several comparison stars allowed variations arising from fluctuations in atmospheric transmission and extinction to be removed. The radioquiet quasars and the reference stars used in the analysis are identified in the CCD images (Fig. 1). Where possible reference stars of comparable brightness and colour to the source were selected for the differential photometry. In all cases two or more reference stars were used. The CCD frames were processed using the standard software package IRAF. The DAOPHOT routine was used to carry out aperture-photometry on each star in the CCD frame. The differential magnitudes were then calculated for any pair of objects in the frame.

\section{Results}

The dates of observations and the differential photometry results for each source are presented in Tables 1 and 2. The source name and redshift are given in Cols. 1 and 2. It should be noted that the right ascension (RA) and declination (DEC) of these sources are included with the finding charts (Fig. 1). The dates when each source was observed and the number of observations in each night per filter are presented in Cols. 3 and 4 respectively. The differential magnitudes $(\Delta B, \Delta V, \Delta R)$ between the reference stars $(R 1, R 2, R 3)$ and the radio-quiet quasar $(Q)$ are given in Col. 5 . In cases where there was more than one observation of a source in a night, the average value of the differential magnitudes are given in Col. 5 . The photometric error $(1 \sigma)$ is given in Col. 6 . 


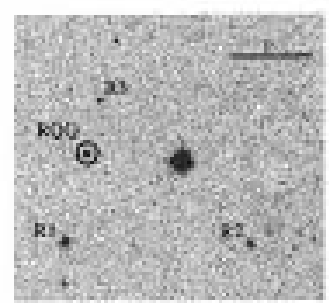

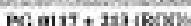

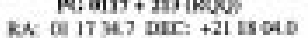

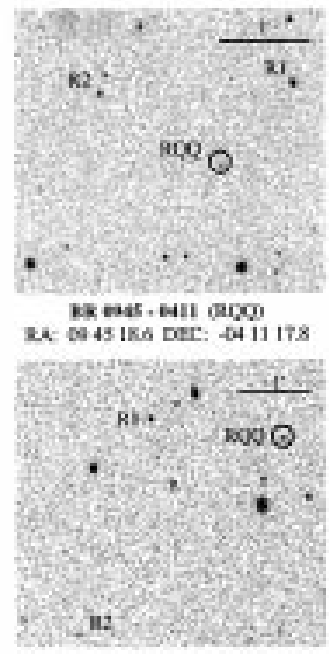

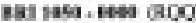

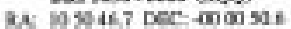

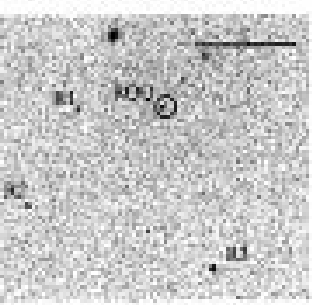

$1145+11100609$
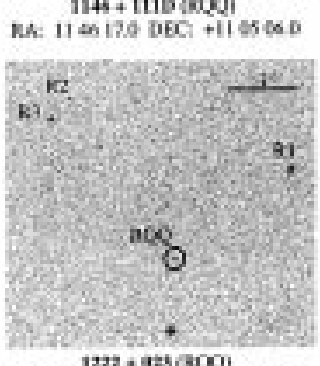

RA. 1t22 $449 \mathrm{DEC}$ +

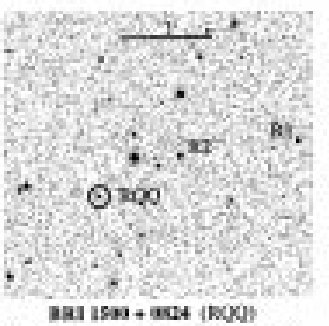

$3931953+504+40004$

RA: 15001898 bEC 4082429.4

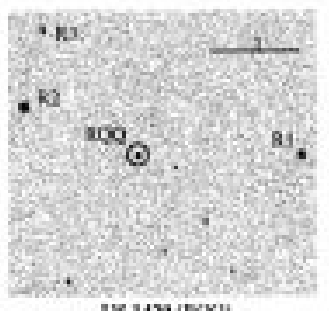

us 1439 (noxul

VA: is 3 is 11.4 bEC: ist 1851 .

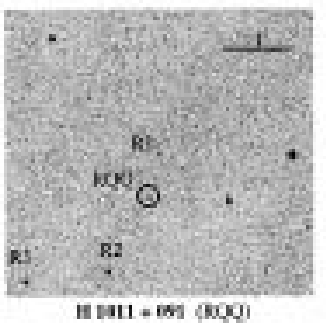

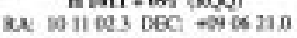

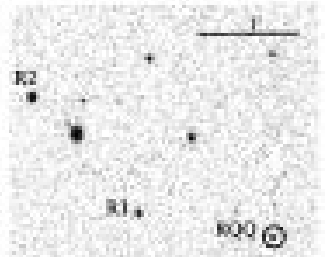

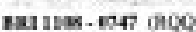
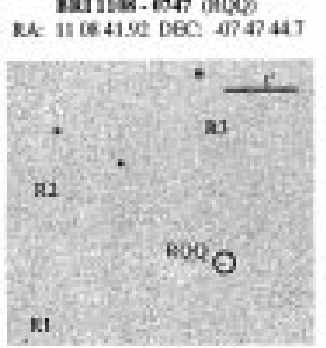

$01159+125(2000)$

RA: 113914.1 Des: +1223120

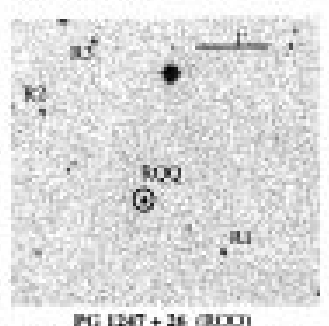

FE $12 \pi T+25$ arom

RA: 1247310 DES: +204700

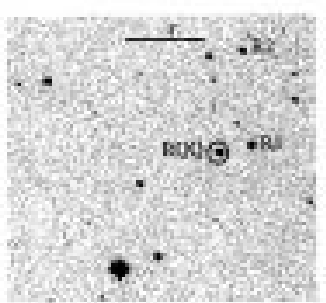

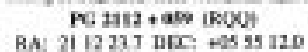
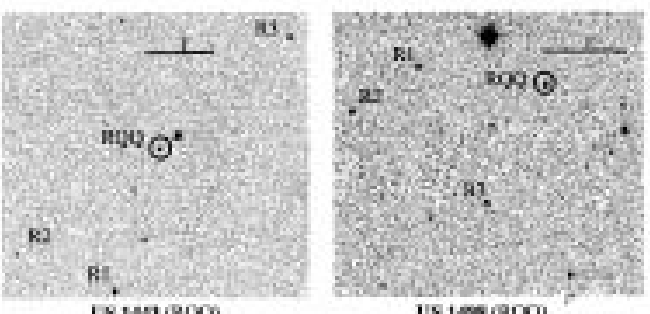

Es taseraco

is iessocon

RA \& 37 a A DRC + in of 970

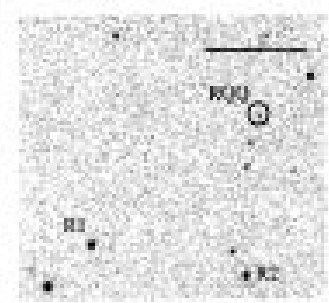

BRI 1ei3 + was (ROO)

R.A. $101314.80 \mathrm{DEC}$ + 0039113

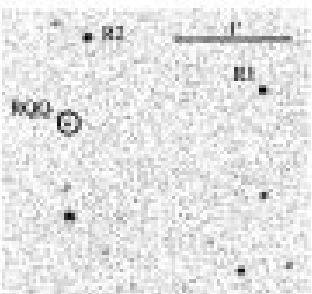

BEI 115a + ales (a.ODI

RA: 111012.3 ver: +010183

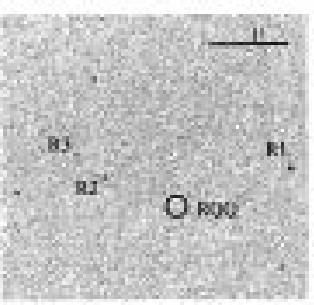

12at-015 $a 000$

U. 12 64 461 DFC Al 31 are

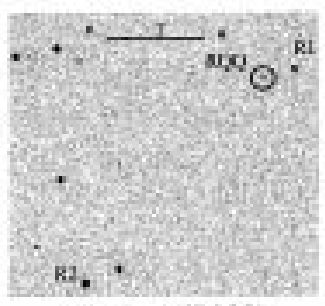

318 1003-4s 23 inger

u. nas3 31.49 bec - 42346.4

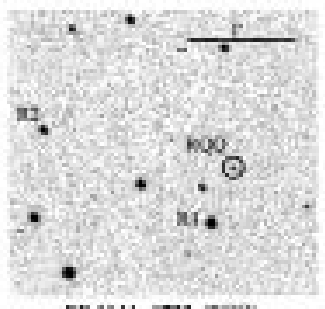

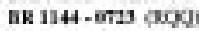

$\mathrm{ua}$ il $440245 \mathrm{DEC}$ - को 23250

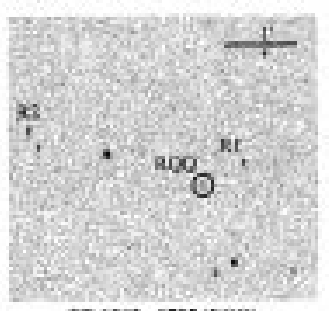

12. $13 n-625$ (100G)

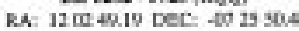
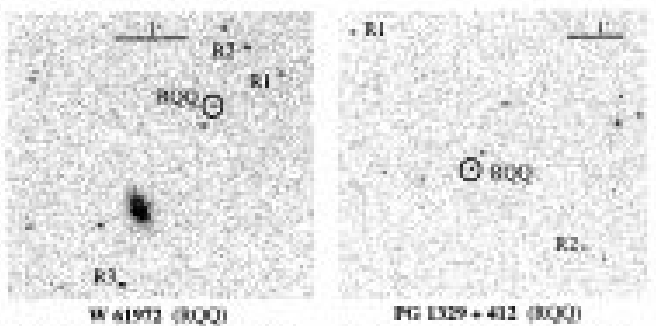

FE $[x s+412$ (oxgon

RA: 132203 DEC +4t in 39

RA. 1258235 DEe + $35 \times 240$

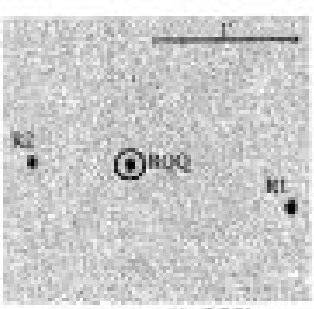

Ni $200+6010000$

Fig. 1. The finding charts for the radio quiet quasars (RQQ) and the reference stars $(R 1, R 2, R 3)$ used in the differential photometry. Each frame is orientated so north is up and east to the left. The length of the bar is one arc minute. B1950 coordinates have been used 

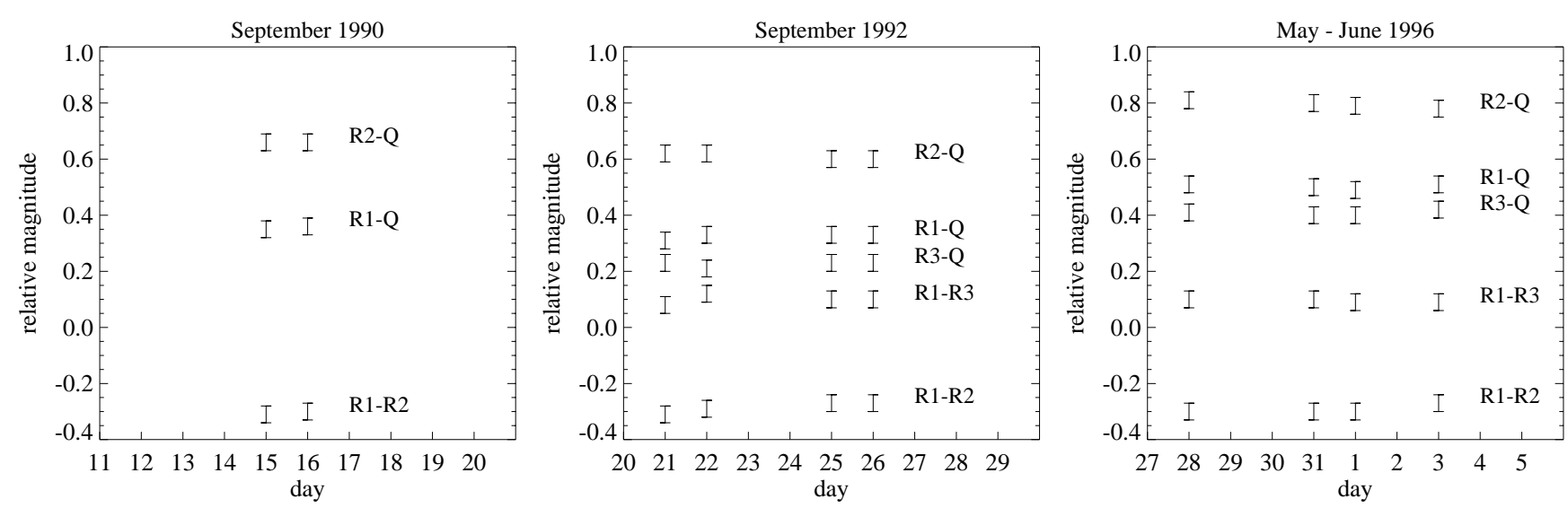

Fig. 2. Differential $V$ band photometry between the radio quiet quasar PG $2112+059$ (QSO) and three reference stars $R 1$, $R 2$ and $R 3$ and differential photometry between the reference stars $R 1-R 2$ and $R 1-R 3$. The star $R 3$ was unobservable during September 1990, due to the small CCD field of view. The average relative magnitude per night is plotted
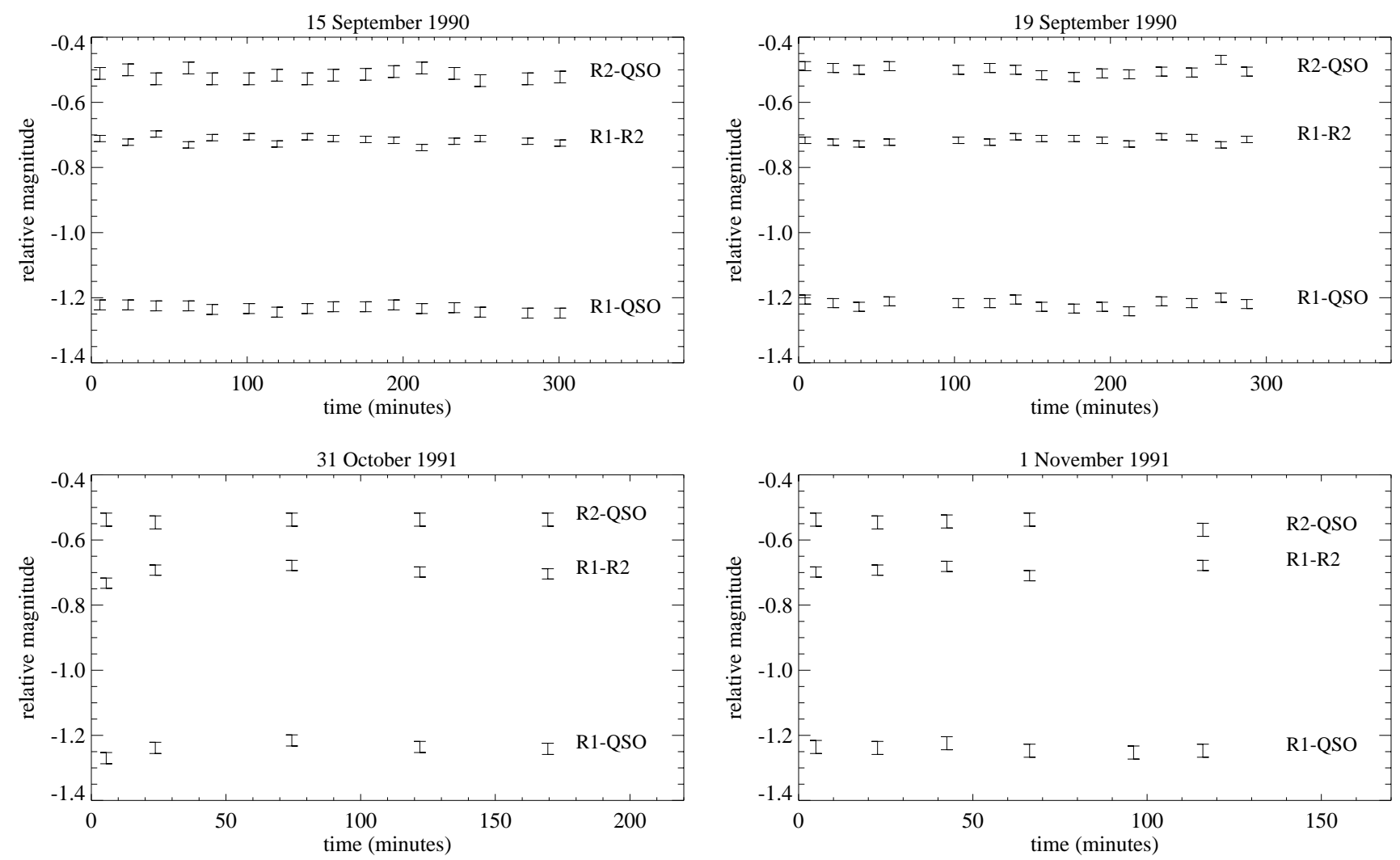

Fig. 3. Differential $V$ band photometry between the radio quiet quasar PG $0117+213$ (QSO) and the two reference stars $R 1$ and $R 2$ and differential photometry between the reference stars $R 1-R 2$. The origins of the time axes of the two upper plots are 00:50 UT on September 15 and 00:32 UT on September 19, 1990. The origins of the time axes of the lower plots are 22:12 UT on October 31 and 22:43 UT on November 1, 1991 
Table 1. $B$ and $V$-band differential photometry results for the three medium redshift radio-quiet quasars

\begin{tabular}{|c|c|c|c|c|c|c|c|c|c|c|c|}
\hline \multirow[b]{2}{*}{ object Name } & \multirow[b]{2}{*}{$z$} & \multirow[b]{2}{*}{$\begin{array}{l}\text { dates of } \\
\text { obs. }\end{array}$} & \multirow{2}{*}{\multicolumn{2}{|c|}{$\begin{array}{l}\text { number of } \\
\text { obs. } \\
\text { per filter }\end{array}$}} & \multicolumn{6}{|c|}{ differential magnitude } & \multirow[b]{2}{*}{$\begin{array}{l}\text { error } \\
1 \sigma\end{array}$} \\
\hline & & & & & $\begin{array}{l}\Delta B \\
R 1-Q\end{array}$ & $R 2-Q$ & $R 3-Q$ & $\begin{array}{l}\Delta V \\
R 1-Q\end{array}$ & $R 2-Q$ & $R 3-Q$ & \\
\hline \multirow[t]{11}{*}{$\overline{\text { PG } 0117+213}$} & 1.493 & $15-09-90$ & 13B & $16 \mathrm{~V}$ & -0.59 & -0.13 & - & -1.23 & -0.52 & - & 0.05 \\
\hline & & 19-09-90 & $14 \mathrm{~B}$ & $15 \mathrm{~V}$ & -0.59 & -0.12 & - & -1.22 & -0.51 & - & 0.05 \\
\hline & & $31-10-91$ & $4 \mathrm{~B}$ & $5 \mathrm{~V}$ & -0.56 & -0.11 & - & -1.23 & -0.53 & - & 0.02 \\
\hline & & 01-11-91 & $5 \mathrm{~B}$ & $6 \mathrm{~V}$ & -0.57 & -0.13 & - & -1.23 & -0.53 & - & 0.02 \\
\hline & & 21-09-92 & $1 \mathrm{~B}$ & $1 \mathrm{~V}$ & -0.57 & - & 3.10 & -1.19 & - & 2.28 & 0.04 \\
\hline & & $22-09-92$ & $1 \mathrm{~B}$ & $1 \mathrm{~V}$ & -0.58 & - & 3.21 & -1.18 & - & - & 0.04 \\
\hline & & 23-09-92 & $1 \mathrm{~B}$ & $1 \mathrm{~V}$ & -0.54 & - & 3.21 & -1.20 & - & - & 0.04 \\
\hline & & 24-09-92 & $1 B$ & $1 \mathrm{~V}$ & -0.56 & - & 3.17 & -1.24 & - & 2.30 & 0.04 \\
\hline & & 25-09-92 & - & $1 \mathrm{~V}$ & - & - & - & -1.23 & - & 2.39 & 0.04 \\
\hline & & 26-09-92 & $1 \mathrm{~B}$ & $1 \mathrm{~V}$ & -0.55 & - & - & -1.21 & - & 2.33 & 0.04 \\
\hline & & 27-09-92 & $1 \mathrm{~B}$ & $1 \mathrm{~V}$ & -0.57 & - & 3.35 & -1.24 & - & 2.32 & 0.04 \\
\hline \multirow[t]{10}{*}{ PG $2112+059$} & 0.466 & $15-09-90$ & - & $8 \mathrm{~V}$ & - & - & - & 0.35 & 0.66 & - & 0.03 \\
\hline & & $16-09-90$ & $6 \mathrm{~B}$ & $6 \mathrm{~V}$ & 0.76 & 1.34 & 一 & 0.35 & 0.65 & - & 0.03 \\
\hline & & 21-09-92 & $2 \mathrm{~B}$ & $2 \mathrm{~V}$ & 0.72 & 1.34 & 0.82 & 0.31 & 0.61 & 0.23 & 0.03 \\
\hline & & 22-09-92 & $1 \mathrm{~B}$ & $1 \mathrm{~V}$ & 0.71 & 1.33 & 0.82 & 0.33 & 0.62 & 0.21 & 0.03 \\
\hline & & 25-09-92 & $2 \mathrm{~B}$ & $2 \mathrm{~V}$ & 0.73 & 1.34 & 0.84 & 0.32 & 0.60 & 0.22 & 0.03 \\
\hline & & 26-09-92 & $1 B$ & $1 \mathrm{~V}$ & 0.72 & 1.32 & 0.82 & 0.33 & 0.60 & 0.23 & 0.03 \\
\hline & & $28-05-96$ & - & $2 \mathrm{~V}$ & - & - & - & 0.51 & 0.81 & 0.41 & 0.03 \\
\hline & & $31-05-96$ & - & $3 \mathrm{~V}$ & - & - & - & 0.50 & 0.80 & 0.39 & 0.03 \\
\hline & & 01-06-96 & - & $2 \mathrm{~V}$ & - & - & - & 0.49 & 0.79 & 0.40 & 0.03 \\
\hline & & 03-06-96 & - & $4 \mathrm{~V}$ & - & - & - & 0.50 & 0.77 & 0.42 & 0.03 \\
\hline \multirow[t]{5}{*}{ PG $2302+029$} & 1.044 & $26-10-91$ & $1 B$ & $1 \mathrm{~V}$ & 0.22 & 0.59 & - & -0.44 & 0.19 & - & 0.03 \\
\hline & & $31-11-91$ & $2 \mathrm{~B}$ & $2 \mathrm{~V}$ & 0.26 & 0.54 & - & -0.40 & 0.24 & - & 0.03 \\
\hline & & 01-11-91 & $1 B$ & $1 \mathrm{~V}$ & 0.25 & 0.56 & - & - & 0.22 & - & 0.03 \\
\hline & & 02-11-91 & $1 \mathrm{~B}$ & $1 \mathrm{~V}$ & 0.25 & 0.60 & - & -0.40 & 0.24 & - & 0.03 \\
\hline & & $20-08-92$ & $1 \mathrm{~B}$ & $1 \mathrm{~V}$ & 0.27 & 0.54 & - & -0.38 & 0.24 & - & 0.03 \\
\hline
\end{tabular}

The major result is that no statistically significant rapid variability was observed for any of the sources listed Tables 1 and 2. The data also reveal no night to night or longer term variability for any of the sources, with the sole exception of PG $2112+059$. This source brightened by 0.18 magnitudes in the $V$-band between September 1992 and June 1996 (Table 1 and Fig. 2). The source with the largest number of observations is PG $0117+213$ (Table 1) and some of the differential $V$-band results are presented in Fig. 3. No significant short term variability was observed. Furthermore careful analysis of the remainder of the $V$-band data and all of the $B$-band revealed no significant short or long term variability.

The sources in this sample can be divided into three main redshift groups; $A(z<2), B(2<z<3), C(z>3)$, and the following section includes comments on a number of the sources in these three categories.

$A(z<2)$ PG $0117+213$ was the most frequently observed source in the sample and no significant variability was detected. The spectra of the source reveal the emission lines CIII and CIV at $z_{\mathrm{em}}=1.493$ (Hewitt \& Burbidge 1987). Infrared observations over a period of years show maximum variability of 0.05 and 0.16 magnitudes at $2.2 \mu \mathrm{m}$ and $10.1 \mu \mathrm{m}$ respectively (Neugebauer et al. 1989). Sagar et al. (1996) observed this RQQSO during November 1996 and detected a hint of optical microvariability and suggested that careful monitoring of this source should continue. The only source in this sample of RQQSOs to display variability is PG 2112+059. It was observed during 1990, 1992 and 1996 and was found to have brightened by 0.18 magnitudes between September 1992 and June 1996 (Table 1). PG $2302+029$ reveals emission peaks in its spectrum which have been identified with FeIII multiplets (Wampler 1986). This source has a redshift $z=1.044$ and $m_{B}=$ 16.03. No variability was detected during the 1991 or 1992 observing runs. US 1420, US 1443 and US 1498 are all ultraviolet excess sources. Spectroscopy of blue and ultraviolet excess sources was reported Mitchell et al. (1984) and the redshifts are given in Table 2. These sources and the remaining sources in this category displayed no rapid variability.

$B(2<z<3) \mathrm{H} 1011+091$ is a broad absorption line (BAL) RQQSO with $m_{V}=17.8$ (Hartig \& Baldwin 1986). The MgII emission line yields $z=2.27$ (Drew $\&$ Boksenberg 1984). The source $1146+111 \mathrm{D}$ has a redshift $z=2.12$ and is part of a compact group of five quasars with similar apparent magnitude and redshift and all within a diameter of 4 arcminutes (Hazard et al. 1979). The sources $1201-015$ and $1222+023$ were 
Table 2. $V$ or $R$-band differential photometry results for twenty high redshift radio-quiet quasars

\begin{tabular}{|c|c|c|c|c|c|c|c|}
\hline \multirow[t]{2}{*}{ object Name } & \multirow[t]{2}{*}{$z$} & \multirow{2}{*}{$\begin{array}{l}\text { dates of } \\
\text { observations }\end{array}$} & \multirow{2}{*}{$\begin{array}{l}\text { number of } \\
\text { observations } \\
\text { per filter }\end{array}$} & \multicolumn{3}{|c|}{ differential magnitude } & \multirow{2}{*}{$\begin{array}{l}\text { error } \\
1 \sigma\end{array}$} \\
\hline & & & & $R 1-Q$ & $R 2-Q$ & $R 3-Q$ & \\
\hline \multirow[t]{4}{*}{ US 1420} & 1.473 & $18-02-93$ & $2 \mathrm{~V}$ & -0.90 & -1.78 & 0.30 & 0.04 \\
\hline & & $20-02-93$ & $1 \mathrm{~V}$ & -0.94 & -1.80 & 0.28 & 0.04 \\
\hline & & $21-02-93$ & $2 \mathrm{~V}$ & -0.94 & -1.82 & 0.34 & 0.04 \\
\hline & & $23-02-93$ & $1 \mathrm{~V}$ & -0.93 & -1.82 & 0.32 & 0.04 \\
\hline \multirow{3}{*}{ US 1443} & 1.564 & $18-02-93$ & $2 \mathrm{~V}$ & -0.97 & -0.74 & -0.52 & 0.03 \\
\hline & & $21-02-93$ & $2 \mathrm{~V}$ & -0.95 & -0.75 & -0.50 & 0.03 \\
\hline & & $23-02-93$ & $2 \mathrm{~V}$ & -0.98 & -0.72 & -0.52 & 0.03 \\
\hline \multirow[t]{3}{*}{ US 1498} & 1.406 & $18-02-93$ & $2 \mathrm{~V}$ & 0.10 & -0.44 & 0.37 & 0.03 \\
\hline & & $21-02-93$ & $2 \mathrm{~V}$ & 0.10 & -0.44 & 0.35 & 0.03 \\
\hline & & $23-02-93$ & $2 \mathrm{~V}$ & 0.09 & -0.45 & 0.34 & 0.03 \\
\hline \multirow[t]{3}{*}{ BR 0945-04 } & 4.118 & $22-02-93$ & $2 \mathrm{R}$ & -3.59 & -2.02 & - & 0.07 \\
\hline & & $24-02-93$ & $2 \mathrm{R}$ & -3.52 & -1.95 & - & 0.03 \\
\hline & & $19-12-95$ & $3 \mathrm{R}$ & -3.54 & -1.97 & - & 0.03 \\
\hline \multirow[t]{3}{*}{ H 1011+091 } & 2.27 & $18-02-93$ & $2 \mathrm{R}$ & - & -1.15 & -1.61 & 0.03 \\
\hline & & $21-02-93$ & $2 \mathrm{R}$ & 0.12 & 1.12 & -1.58 & 0.03 \\
\hline & & $23-02-93$ & $2 \mathrm{R}$ & 0.11 & -1.10 & -1.60 & 0.03 \\
\hline \multirow[t]{2}{*}{ BRI 1013+00 } & 4.38 & $22-02-93$ & $2 \mathrm{R}$ & -2.76 & -2.89 & - & 0.03 \\
\hline & & $24-02-93$ & $2 \mathrm{R}$ & -2.78 & -2.89 & - & 0.03 \\
\hline BR 1033-03 & 4.50 & $22-02-93$ & $2 \mathrm{R}$ & -1.13 & -2.59 & - & 0.03 \\
\hline \multirow[t]{2}{*}{ BRI 1050-00 } & 4.29 & $22-02-93$ & $2 \mathrm{R}$ & -0.37 & 0.20 & - & 0.04 \\
\hline & & $19-12-95$ & $2 \mathrm{R}$ & -0.32 & 0.22 & - & 0.04 \\
\hline BRI 1108-07 & 3.94 & $22-02-93$ & $2 \mathrm{R}$ & -0.38 & -1.83 & - & 0.04 \\
\hline BRI 1110+01 & 3.93 & $22-02-93$ & $2 \mathrm{R}$ & -1.67 & -1.87 & - & 0.04 \\
\hline BR 1144-08 & 4.16 & $22-02-93$ & $2 \mathrm{R}$ & -2.60 & -1.83 & - & 0.04 \\
\hline \multirow[t]{4}{*}{$1146+111 D$} & 2.12 & $18-02-93$ & $2 \mathrm{R}$ & -0.20 & 0.15 & -2.03 & 0.03 \\
\hline & & $20-02-93$ & $2 \mathrm{R}$ & -0.20 & 0.13 & -2.04 & 0.03 \\
\hline & & $21-02-93$ & $2 \mathrm{R}$ & -0.18 & 0.17 & -2.00 & 0.03 \\
\hline & & $23-02-93$ & $2 \mathrm{R}$ & -0.19 & 0.18 & -1.99 & 0.03 \\
\hline \multirow[t]{3}{*}{$1159+123$} & 3.51 & $18-02-93$ & $2 \mathrm{R}$ & 0.23 & -0.29 & 0.44 & 0.04 \\
\hline & & $20-02-93$ & $2 \mathrm{R}$ & 0.21 & -0.28 & 0.47 & 0.04 \\
\hline & & $21-02-93$ & $2 \mathrm{R}$ & 0.20 & -0.29 & 0.44 & 0.04 \\
\hline \multirow[t]{3}{*}{$1201-015$} & 2.26 & $19-02-93$ & $2 \mathrm{R}$ & -3.39 & -2.38 & - & 0.04 \\
\hline & & $21-02-93$ & $1 \mathrm{R}$ & -3.44 & -2.34 & -0.68 & 0.04 \\
\hline & & $23-02-93$ & $2 \mathrm{R}$ & -3.45 & -2.33 & -0.67 & 0.04 \\
\hline BR 1202-07 & 4.70 & $22-02-93$ & $2 \mathrm{R}$ & -0.03 & -0.61 & - & 0.07 \\
\hline \multirow[t]{3}{*}{$1222+023$} & 2.05 & $19-02-93$ & $2 \mathrm{R}$ & -3.00 & 0.92 & -1.81 & 0.05 \\
\hline & & $21-02-93$ & $2 \mathrm{R}$ & -3.02 & 0.92 & -1.81 & 0.05 \\
\hline & & $23-02-93$ & $2 \mathrm{R}$ & -2.98 & 0.93 & -1.76 & 0.05 \\
\hline \multirow[t]{2}{*}{ PG $1247+268$} & 2.041 & $18-02-93$ & $1 \mathrm{R}$ & 1.37 & 2.03 & 1.73 & 0.03 \\
\hline & & $21-02-93$ & $2 \mathrm{R}$ & 1.35 & 2.06 & 1.77 & 0.03 \\
\hline \multirow[t]{3}{*}{ W 61972} & 1.92 & $18-02-93$ & $2 \mathrm{R}$ & 0.51 & -1.20 & -1.83 & 0.02 \\
\hline & & $21-02-93$ & $2 \mathrm{R}$ & 0.51 & -1.20 & -1.82 & 0.02 \\
\hline & & $23-02-93$ & $1 \mathrm{R}$ & - & -1.20 & -1.85 & 0.02 \\
\hline \multirow[t]{2}{*}{ PG $1329+412$} & 1.93 & $18-02-93$ & $2 \mathrm{R}$ & -0.67 & 0.79 & -1.55 & 0.03 \\
\hline & & $23-02-93$ & $2 \mathrm{R}$ & -0.67 & 0.77 & -1.54 & 0.03 \\
\hline \multirow[t]{2}{*}{ BRI $1500+08$} & 3.96 & $23-02-93$ & $2 \mathrm{R}$ & -0.82 & -0.92 & - & 0.07 \\
\hline & & $17-03-94$ & $2 \mathrm{R}$ & -0.86 & - & - & 0.07 \\
\hline
\end{tabular}

detected optically and listed by MacAlpine \& Williams (1981). 1201-015 has an estimated $m_{V}=18.0$, and emission lines $\lambda 3970$ and $\lambda 4560$ believed to be Ly $\alpha$ and OIv yielding $z=2.26 ; 1222+023$ has $z=2.05$ and an estimated $m_{V}=17.0$. PG $1247+268$ was observed by Green et al. (1980). The optical spectrum shows four strong emission features Ly $\alpha+\mathrm{NV}, \mathrm{SiIV}+\mathrm{OIV}, \mathrm{CIV}$ and CIII with $z=2.041$ and $m_{V}=15.8$.

$C(z>3) 1159+123$ discovered by Hazard et al. (1984) using objective prism plates from the UK Schmidt telescope is a strong emission-line RQQSO with $z_{\mathrm{em}}=$ 3.51 and $m_{V}=17.5$. Irwin et al. (1991) carried out 
a very successful multicolour survey in $B, R$ and $I$ (selected $B-R$ ) to search for high redshift quasars. Using this method they found 27 quasars at $z>4$. $R$ band data for nine of these high redshift quasars are given in Table 2: $B R$ 0945-0411, $m_{R}=18.80 ; B R I$ $1013+0035, m_{R}=18.80 ; B R 1033-0327, m_{R}=18.50$; $B R I$ 1050-0000, $m_{R}=18.59 ; B R I 1108-0747, m_{R}=$ $18.13 ; B R I 1110+0106, m_{R}=18.30 ; B R$ 1144-0723, $m_{R}=18.60 ; B R 1202-0725, m_{R}=18.70$ and $B R I$ $1500+0824, m_{R}=19.25$. No variability was found, however the search for optical variability in these high redshift RQQSOs will continue.

\section{Discussion}

The mechanisms that make some quasars radio-loud and others radio-quiet are not well understood but the major differences may be attributable to the spin of the black hole (Wilson \& Colbert 1995). There are a number of well established distinctions between the two classes. (i) Radio-loud quasars are associated with elliptical host galaxies and radio-quiet quasars tend to reside in spiral galaxies. The mean absolute magnitude of the underlying galaxies of radio-loud quasars is similar to that of radio galaxies, however the host galaxies of radio-quiet quasars are $0.6-1.0$ magnitudes less luminous (Smith et al. 1986; Véron-Cetty \& Woltjer 1990). Recent deep near infrared ( $K$-band) imaging of host galaxies of quasars revealed that more than half of RQQSOs appear to lie in galaxies that are dominated by an exponential disk (Taylor et al. 1996). Those RQQSOs that have elliptical host galaxies show signs of interaction and are in general more luminous than those that reside in disk galaxies. There is also some evidence to suggest that a large majority of low-luminosity radio-quiet AGN lie in disk galaxies but a significant fraction of RQQSOs more luminous than $M_{V} \approx-23.5$ have elliptical host galaxies. (ii) Unlike RQQSOs, radio-loud quasars produce large scale jets and lobes and can be defined by their radio luminosity those with $L(5 \mathrm{GHz})<10^{25} \mathrm{~W} \mathrm{~Hz}^{-1} \mathrm{sr}^{-1}$ are classified as RQQSOs, and those with $L(5 \mathrm{GHz})>10^{25} \mathrm{~W} \mathrm{~Hz}^{-1} \mathrm{sr}^{-1}$ as radio loud quasars (Miller et al. 1990). (iii) Gammaray results recently obtained by the Compton Gamma Ray Observatory (CGRO) have produced evidence for two classes of AGN (Dermer \& Gehrels 1995). These classes are defined by their redshift, luminosity distributions and high energy spectral properties. The first class of objects have redshifts $\leq 0.06$ and $50-150 \mathrm{keV}$ luminosities in the range $10^{41}-10^{44} \mathrm{ergs}^{-1}$. Associated with this group are Seyfert galaxies, RQQSOs and radio-galaxies viewed at large angles with respect to the radio jet axis. The gammaray spectra for these sources soften between $\sim 100 \mathrm{keV}$ and several MeV. The second class of source consists of blazars with redshifts as large as 2.3 which have detectable fluxes of gamma-rays above $100 \mathrm{MeV}$ with emission extending into the $\mathrm{GeV}$ band. This class of source probably consists of AGN that are observed close to the axis of a radio jet. So far no RQQSO has been detected at gamma ray energies above $10 \mathrm{MeV}$.

Unlike radio-loud quasars, intensive optical monitoring of RQQSOs only started in the early 1990s. GopalKrishna et al. (1995) monitored a sample of six optically bright and luminous RQQSOs $\left(m_{V} \approx 16\right.$ and $\left.M_{V}<-23\right)$ and found strong hints for variability in three RQQSOs; PG $0946+301$ displayed an $R$-band increase of $\sim 0.05$ magnitudes in a time of $\sim 0.5 \mathrm{hr}$; PG 1049-006 varied in $V$-band by $\sim 0.05$ magnitudes in $\sim 0.6 \mathrm{hr}$ and $\mathrm{PG}$ $1206+459$ varied by 0.04 magnitudes in $\sim 2 \mathrm{hr}$ in the $R$-band. Sagar et al. (1996) reported that the flux density from PG $1444+407$ dropped by 0.04 magnitudes in $\sim 0.5 \mathrm{hr}$ in the $R$-band. Sagar et al. (1996) also reported long-term variability (over $\sim 1 \mathrm{yr}$ ) for four RQQSOs in their sample, with the largest variability of about 0.15 magnitudes in 11 months, recorded for the source PG 1049-005. Jang \& Miller (1995) reported intranight variability in the RQQSO II Zw 175 which varied by $\sim 0.05$ magnitudes over a period of 4 days. The reported detection of variability in RQQSOs is not in conflict with the results presented here and in Tables 1 and 2, because the small levels of variability would not have been detected above the $3 \sigma$ level in many of the sources monitored in this survey. One source, PG 2112+059, displayed longterm variability decreasing by 0.18 magnitudes in the $V$ band over a period of almost four years.

The long term variability of large samples of opticallyselected quasars have been studied over decades (Hook et al. 1994; Cristiani et al. 1996). In these samples, a strong negative correlation between variability and quasar luminosity was found with the more luminous quasars displaying less variability. This result is interesting considering that RQQSOs in this sample all have high luminosities $\left(-27<M_{V}<-30\right)$, and no rapid optical or night to night variability was detected in any of the sources.

\subsection{Radio results on $R Q Q S O s$}

The radio spectra of RQQSOs probably have contributions from three components: (i) optically thin synchrotron from star forming regions in the disk of the host galaxy and in a circumnuclear starburst, (ii) optically thin synchrotron from an extended or possibly jet-like component powered by an active nucleus and (iii) partially opaque synchrotron from a compact VLBI-scale core. In an extensive survey of RQQSOs, Kellermann et al. (1994) obtained VLA maps which show extended and double lobe radio structures in some sources that are similar to those observed in radio-loud quasars. The RQQSOs mostly have a radio luminosity well in excess of the $10^{22} \mathrm{~W} \mathrm{~Hz}^{-1}$ found for most normal spiral and elliptical galaxies and hence the radio emission is not simply that from the underlying galaxy. It is quite possible that for sensitivity reasons quasars with additional low-surface brightness 
features may have been missed in the VLA mapping of the radio-quiet sources. Nevertheless, a number of the radioquiet quasars in the Kellermann et al. (1994) survey (e.g. $0953+41 ; 1116+21 ; 1634+70)$ are well resolved and show extended structure ranging between 49 and $\sim 300 \mathrm{kpc}$. Recent VLA results (Barvainis et al. 1996) revealed heterogeneous spectral shapes in the radio spectra of a sample of RQQSOs that could be classified into general categories similar to radio loud quasars. Furthermore variability was discovered for seven sources most of which had flat or inverted radio spectra. In one source VLBI revealed that essentially all the flux emanated from one compact subparsec core. The radio results on these types of sources appear to be inconsistent with starburst models and imply that the cores of many RQQSOs may be scaled down versions of those found in radio loud quasars (Stein 1996).

\subsection{The remarkable RQQSOs, IRAS $13349+2438$ and $P G 1416-129$}

Recent new results have highlighted the unusual properties of two RQQSOs, both of which lie near the top of the upper band of radio emission for RQQSOs $(R \sim$ $1)$. The first source IRAS $13349+2438$ was initially detected through its strong infrared emission (Beichman et al. 1986) and has been classified as a radio-quiet, infrared bright quasar with a value of $R=1.9$. It has a redshift of $z=0.107$ and a high polarisation which rises from $1.4 \%$ at $2.2 \mu \mathrm{m}$ ( $K$-band) to $8 \%$ at $0.36 \mu \mathrm{m}$ ( $U$-band). Wills et al. (1992) found no variability of the polarisation or flux density on timescales from days to months and discussed a bipolar geometry to account for its polarisation and other properties. VLA observations revealed an unresolved source with an unusual radio spectrum with a maximum flux density of $7 \mathrm{mJy}$ at a frequency of $5 \mathrm{GHz}$. The origin of the peaked radio emission is not understood but absorption of the radio emission may occur in the dusty dense interstellar medium and also the contributions of different spectra from several source components may be involved.

IRAS $13349+2438$ has high polarisation, strong FeII emission and is radioquiet but no broad absorption lines (BAL) have been observed. IRAS $13349+2438$ has been observed on several occasions by ROSAT where the source was found to vary by a factor of 4.1 in about one year and about $25 \%$ in one week (Brandt et al. 1996) but showed no evidence for the large intrinsic absorption of soft X-rays by cold neutral matter. The soft X-ray variability excluded electron scattering for most of the soft X-rays and suggest absorption by a warm ionized gas with internal dust.

Recent ASCA observations of IRAS $13349+2438$ discovered for the first time rapid X-ray variability or blazar like behaviour in a RQQSO. The source displayed intensity variations on two separate occasions by factors of two on timescales of only a few hours without any significant spectral changes (Brinkmann et al. 1996). There is also some evidence in the X-ray data for even more rapid variability. The 0.6 to $8 \mathrm{keV}$ spectrum was fitted with a power law with $\Gamma=2.40$ which is steeper than the average value of $\Gamma=1.9$ found for RQQSOs in the ASCA band. Brinkmann et al. (1996) point out that the line of sight to the quasar may graze the edge of the torus and suggest that small changes in viewing conditions could produce marked changes in intensity and spectral shape. However it is difficult to understand how changes in viewing conditions could produce such rapid variability with no significant spectral changes. It is plausible that IRAS $13349+2438$ is an example of a radio loud quasar that is viewed through a dusty ionizing outflow, possibly associated with a merger, that severely attenuates the radio emission so that the source is classified as a RQQSO. In this context it should be noted that the quasar III $\mathrm{Zw}$ 2 could be classified as a RQQSO at $5 \mathrm{GHz}$ but the radio spectrum rises steeply toward higher frequencies and this source is radio-loud when the $90 \mathrm{GHz}$ flux density is adopted (Schnopper et al. 1978). Further observations of IRAS $13349+2438$ across the full spectrum including VLBI searches for a compact self-absorbed component or indeed multiple source components may help elucidate the nature of this unusual hybrid type source.

The second unusual RQQSO is PG 1416-129 at $z=0.129$. It has been classified (Turnshek \& Grillmair 1986; Ulrich 1988) as a broad absorption line quasar (BALQSO). The value of $R$ is 1.1 and similar to IRAS $13349+2438$ it lies near the top of the upper band for RQQSOs which is also heavily populated with BALQSOs (Francis et al. 1995). This source has a soft X-ray excess (de Kool \& Meurs 1994) and the hardest spectral index of any source in the energy range $2-20 \mathrm{keV}$ when observed with GINGA (Williams et al. 1992). Staubert \& Maisack (1996) detected this bright RQQSO at energies above $50 \mathrm{keV}$ with the OSSE telescope on CGRO and the flux was found to be variable on a timescale of days during the 14 day observation.

The BAL classification of this source has been questioned by Green \& Mathur (1996) who proposed that large values of the optical to X-ray slope $\left(\alpha_{\text {ox }}>1.8\right)$ be the defining characteristic of BALQSOs. They report a low value of $\left(\alpha_{\mathrm{ox}} \sim 1.4\right)$ for $\mathrm{PG} 1416-129$ and suggest further observations with HST to check the BAL classification. VLA observations of PG 1416-129 reveal unusual results for a RQQSO: (i) the radio source consists of an unresolved core coincident with an extended component that is assumed not to be an unrelated background source (Kellermann et al. 1994), (ii) the source varied by a factor of 4.5 over a period of ten years and could have been more variable given the very limited monitoring at radio frequencies and (iii) the radio spectrum is consistent with a flat or inverted spectrum (Barvainis et al. 1996). No VLBI observations have been reported for PG 1416-129 but one of the other variable RQQSOs PG $1216+069$ was detected with essentially all of its flux in the VLBI core 
and the high brightness temperature limit confirmed the self-absorbed synchrotron hypothesis for the flat spectrum component (Barvainis et al. 1996). This component may well be a scaled-down version of the radio sources observed in radio loud quasars and future VLBI observations of $\mathrm{PG}$ 1416-129 may also reveal a similar component.

\section{Conclusions}

A long-term survey of a sample of high luminosity $(-27<$ $\left.M_{V}<-30\right)$ and medium to high redshift $(0.466<z<$ $4.7)$ radio-quiet quasars was undertaken, in order to search for short and long term optical variability on timescales of hours to years. A large sample of 23 RQQSOs was observed, with a total observation time of about 60 hours spread over a period of several years. Long-term variability was detected in the RQQSO PG $2112+059$ when it varied by 0.18 magnitudes in the $V$-band between 1992 and 1996. No rapid variability was observed in any of the sources in this sample of RQQSOs. The finding charts are included because they identify the RQQSO and the reference stars used in the photometry and hence are available to other observers.

There have been a few reports of rapid optical variability in a number of RQQSOs but these reports are not in conflict with the results presented here because such small variability would not have been detected in many of the sources monitored in this survey. The unusual properties of two sources are highlighted. These sources were not monitored in this survey but have recently been added to the list of sources for study. The remarkable source IRAS $13349+2438$ combines some of the properties of blazars and radio quiet quasars and hence further observations may elucidate the nature of this hybrid source. The two unusual sources have $R$ values near the top of the range for RQQSOs and also have unusual radio spectra that may signify the presence of several source components. Further observations with VLA and VLBI should reveal new and enlightening views on the radio properties of these sources.

Acknowledgements. The Jacobus Kapteyn Telescope on the island of La Palma is operated by the Royal Greenwich Observatory at the Spanish Observatorio del Roque de los Muchachos of the Institato de Astrofisica de Canarias. We are grateful to Catherine Handley and Matt Delaney for their help in the preparation of this paper.

\section{References}

Abramowicz M.A., Bao G., Lanza A., Zhang X.H., 1991, A\&A 245,454

Barvainis R., Lonsdale C., Antonucci, 1996, AJ 111, 1431
Beichman C.A., Soifer B.T., Helou G., et al., 1986, ApJ 308, L1

Brandt W.N., Fabian A.C., Pounds K.A., 1996, MNRAS 278, 326

Brinkmann W., Kawai N., Ogasaka Y., Siebert J., 1996, A\&A 316, L9

Cristiani S., Trentini S., La Franca F., et al., 1996, A\&A 306, 395

Crusius A., Schlickeiser R.A., 1988, A\&A 196, 327

de Kool M., Meurs E.J.A., 1994, A\&A 281, L65

Dermer C.D., Gehrels N., 1995, ApJ 447, 103

Drew J.E., Boksenberg A., 1984, MNRAS 211, 813

Francis P.J., Hooper E.J., Impey C.D., 1993, AJ 106, 417

Gopal-Krishna, Subramanian K., 1991, Nat 349, 766

Gopal-Krishna, Sagar R., Wiita P.J., 1995, MNRAS 274, 701

Gopal-Krishna, Wiita P.J., Altieri B., 1993, A\&A 271, 89

Green R.F., Pier J.R., Schmidt M., et al., 1980, ApJ 239, 483

Green P.J., Mathur S., 1996, ApJ 462, 637

Hartig G.F., Baldwin J.A., 1986, ApJ 302, 64

Hazard C., Arp H.G., Morton D.C., 1979, Nat 282, 271

Hazard C., Terlevich R., McMahon R., et al., 1984, MNRAS $211,45 p$

Hewitt A., Burbidge G., 1987, ApJS 63, 1

Hook I.M., McMahon R.G., Boyle B.J., Irwin M.J., 1994, MNRAS 268, 305

Hughes P.A., Aller H.D., Aller M.F., 1986, ApJ 341, 68

Irwin M.J., McMahon R.G., Hazard C., 1991, in: The Space Distribution of Quasars, Crampton D. (ed.) APS Conf. Ser. 21,117

Jang M., Miller H.R., 1995, ApJ 452, 582

Kellermann K.I., Sramek R.A., Schmidt M., Shaffer D.B., Green R., 1989, AJ 98, 1195

Kellermann K.I., Sramek R.A., Schmidt M., Green R., Shaffer D.B., 1994, AJ 108, 1163

MacAlpine G.M., Williams G.A., 1981, ApJS 45, 113

Mangalam A.V., Witta P.J., 1993, ApJ 406, 420

Marscher A.P., 1980, ApJ 239, 296

Mathur S., Elvis M., Singh K.P., 1995, ApJ 455, L9

McBreen B., Metcalfe L., 1987, Nat 330, 348

Miller L., Peacock J.A., Mead A.R.G., 1990, MNRAS 244, 207

Mitchell K.J., Warnock III A., Usher P.D., 1984, ApJ 287, L3

Neugebauer G., Soifer B.T., Matthews K., Elias J.H., 1989, AJ 97, 957

Pica A.J., Smith A.G., Webb J.R., et al., 1988, AJ 96, 1215

Qian S.J., Quirrenbach A., Witzel A., et al., 1991, A\&A 241, 15

Rabbette M., McBreen B., Steel S., Smith N., 1996, A\&A 310, 1

Romero G.E., Surpi G., Vucetich H., 1995, A\&A 301, 641

Sagar R., Gopal-Krishna, Wiita P.J., 1996, MNRAS 281, 1267

Schnopper H.W., Delvaille J.P., Epstein J.P., et al., 1978, ApJ 222, L91

Smith E.P., Heckman T.M., Bothun G.D., Romanishin W., Balick B., 1986, ApJ 306, 64

Sopp H.M., Alexander, P., 1991, MNRAS 251, 14p

Staubert R., Maisack M., 1996, A\&A 305, L41

Stein W.A., 1996, AJ 112, 909

Storrie-Lombardi L.J., McMahon R.G., Irwin M.J., 1996, ApJ 468, 121

Taylor G.L., Dunlop J.S., Hughes P.H., Robson E.I., 1996, MNRAS 283, 930 
Terlevich R., Tenorio-Tagle G., Franko J., Melnick J., 1992, MNRAS 255, 713

Turnshek D.A., Grillmair C.J., 1986, ApJ 310, L1

Ulrich I.M.H., 1988, MNRAS 230, 121

Usher P.D., 1981, ApJS 46, 117

Véron-Cetty M.-P., Véron P., 1985, A Catalogue of Quasars and Active Galactic Nuclei, 2nd ed., Publ. ESO

Véron-Cetty M.-P., Woltjer L., 1990, A\&A 236, 69

Wampler E.J., 1986, A\&A 161, 223
Webb J.R., Smith A.G., Leacock R.J., et al., 1988, AJ 95, 374 Wiita P.J., Miller H.R., Gupta N., Chakrabarti S.K., 1992, in: Variability of Blazars, Valtaoja E., Valtonen M. (eds.). Cambridge Univ. Press, Cambridge, p. 311

Wills B.J., Wills D., Evans II N.J., et al., 1992, ApJ 400, 96

Williams O.R., Turner M.J.L., Stewart G.C., Saxton R.D., et al., 1992, ApJ 389, 157

Wilson A.S., Colbert E.J.M., 1995, ApJ 438, 62 